\title{
The pattern of growth hormone secretion during the menstrual cycle in normal and depressed women
}

\author{
Josephine Z. Kasa-Vubu*, Eleni V. Dimaraki† and Elizabeth A. Young‡ \\ ${ }^{\star}$ Departments of Pediatrics, $\dagger$ Internal Medicine and $\ddagger$ Psychiatry, University of Michigan, Ann Arbor Michigan
}

\section{Summary}

Objective Major depression is associated to altered hypothalamicpituitary function. Stress is linked to elevated cortisol as well as menstrual cycle disturbance; however, there is no known relationship between depression and menstrual cycle disruption. The aim of this study was to investigate changes of growth hormone $(\mathrm{GH})$ secretion during the menstrual cycle in normal and depressed women.

Design Case-control study.

Patients and methods Nineteen women affected with depression and 24 normal controls were included. The two groups had comparable body mass index (BMI), and age (29.4 $\pm 9 \cdot 8$ vs. $28 \cdot 6 \pm 9 \cdot 7$ years). Nine depressed and 10 controls were studied in the follicular phase, while 10 depressed and 14 controls were studied in the luteal phase of the cycle. GH was sampled every $10 \mathrm{~min}$ for $24 \mathrm{~h}$, and the data were analysed by the cluster pulse detection method.

Results There was no difference in 24-h mean GH concentrations between depressed and control subjects $(P=0.93)$, even after accounting for menstrual cycle phase $(P=0 \cdot 38)$. GH pulse frequency was higher during the follicular phase of the cycle $(P=0.032)$, and nocturnal GH was higher in the follicular phase of the cycle ( $P=0 \cdot 05$, and after adjusting for 24-h GH, $P=0 \cdot 0138$ ) regardless of whether the subjects were depressed or healthy.

Conclusions In studies of GH secretion in women with or without depression, it is necessary to control for the phase of menstrual cycle.

(Received 12 January 2005; returned for revision 26 January 2005; finally revised 21 February 2005; accepted 9 March 2005)

Major depression is accompanied by abnormalities in multiple endocrine systems. Depression alters pituitary function by increasing

This study was funded by grant MH 50030-04 and K05 MH01931 (EAY), 1K12HD01438-01 BIRCWH from the Office of Women's Health Research (JZKV) and the National Institute of Child Health, 1 K23 DK065995(JZKV), and was supported by a General Clinical Research Center Grant M01-RR-0042.

Correspondence: Josephine Z. Kasa-Vubu, MD, MS, Department of Pediatrics, Division of Pediatric Endocrinology, University of Michigan Medical Center, Medical Professional Building Room 1205/0718, Ann Arbor, MI 48019-0718. Fax: 734-615-3353; E-mail: jzkv@med.umich.edu the secretion of ACTH and cortisol. Whereas there is no known relationship between depression and menstrual cycle disruptions, ${ }^{1}$ stress, which is associated with elevated cortisol concentrations, is linked to disturbances of menstrual cyclicity. ${ }^{2}$ Abnormal regulation of thyroid hormones, driven by another pituitary-regulated hormonal axis, is also associated with depressive symptoms. ${ }^{3,4}$ While acute stress increases GH release, chronic stress is associated with a decrease of growth hormone secretion. ${ }^{5}$ Conditions with endogenous cortisol excess, such as Cushing's syndrome, are associated with suppressed GH concentrations, ${ }^{6}$ as well as symptoms of depression. ${ }^{7}$ However, it is not known whether depression, occasionally associated with chronic hypercortisolemia, ${ }^{8,9}$ also alters GH concentrations. Previous studies on GH secretion dynamics in depression were not sex-matched, ${ }^{10}$ did not take into account the phases of the menstrual cycle, ${ }^{11}$ or had limited GH sampling paradigms. ${ }^{12}$ The possibility of altered GH secretory dynamics in depression is supported by reports that in affected subjects, there is blunting of GH response to pharmacological agents used in provocative diagnostic tests when compared to normal controls. $10,11,13-15$

Growth hormone patterns of secretion in women are distinct from that of male subjects. In women, growth hormone secretory patterns have less day-night variation than in men, ${ }^{16}$ but whether GH varies across the menstrual cycle phase is less clear. ${ }^{17}$ We sought to determine whether depression would be associated with a decrease in GH in depressed premenopausal women recruited in both the follicular and the luteal phases of the menstrual cycle compared to normal controls.

\section{Subjects and methods}

\section{Subjects}

All participants were premenopausal women 20-50 years of age, who represent a subset from a larger group previously described, ${ }^{18}$ and from whom samples were still available. Depressed women were recruited from patients presenting to the University of Michigan Mood Disorders Program for treatment. We used an unselected patient population, including patients with atypical depression rather than restricting our population to melancholic depressed women only. The original study from which this study represents a subset, included 26 pairs of control and depressed women with Hamilton ratings for severity of depression of $1.0 \pm 1 \cdot 5$ and $19 \cdot 6 \pm 5 \cdot 3$, respectively. ${ }^{19}$ We measured $\mathrm{GH}$ in 24 controls and 19 affected 
women. The difference in the size of the two groups was a result of insufficient volume to perform GH in some depressed participants; in addition, one subject with only 12 -h sampling was eliminated from the initial pool of subjects.

All studies were approved by the University of Michigan Institutional Review Board (IRB), and all participants gave written informed consent. All subjects were otherwise healthy, and untreated for the current episode at the time of the study except for one treatment refractory depressed patient who was studied on antidepressant therapy, and did not change the overall results. All others were free of psychotropics and any other medications or hormonal contraception except for nonprescription pain medications, for more than 3 months. Exclusion criteria also included shift work of travel over more than (three) time zones within 3 months prior to enrolment. The study was conducted in the General Clinical Research Center (GCRC), where subjects were admitted for $26 \mathrm{~h}$. A Structured Clinical Interview for Diagnostic and Statistical Manual of Mental Disorders (DSM-IV) Axis 1 disorders (SCID-IV) ${ }^{20}$ interview and structured Hamilton rating were conducted on all patients by our research nurse to confirm the diagnosis. Normal controls had no previous psychiatric diagnosis as confirmed by Structured Clinical Interview for DSM III-R (SCID-NP) ${ }^{21}$ conducted by our research nurse. They had no first degree relative with any Axis I disorder, and no second degree relative with an anxiety or mood disorder as ascertained by questioning the subject on all relatives. All subjects underwent a screening physical exam, blood work and urine drug screen. Controls were recruited to match depressed patients based on age, BMI, menstrual cycle day and length. Each subject was studied once in either the follicular or the luteal phase of the cycle. The menstrual cycle phase was further confirmed with 24-h patterns for luteinizing hormone, and progesterone determination. ${ }^{19}$

Protocol. Subjects presented to the CRC at 8:00 h on day 1 , when an intravenous catheter was inserted. After a 60 -min recovery period, 10-min sampling was initiated for $24 \mathrm{~h}$. Normal saline was infused throughout the study. While subjects were able to get up to go to the bathroom, they remained at bed rest for the 24-h duration of the study. Standardized meals were served at set times. Eating between meals was not permitted, but decaffeinated beverages were provided at set times. Subjects were free to turn off the lights at their usual sleep times, but all lights were off by midnight. During overnight sampling, blood was drawn with the aid of a flashlight, and nurses recorded whether the subjects were awake or asleep with each 10 -min sample. All samples were frozen at $-80{ }^{\circ} \mathrm{C}$ until assayed for growth hormone.

Hormone Assays. Plasma GH concentrations were measured using a chemiluminescent enzyme immunometric assay for use with the Immulite Automated Analyser (Immulite ${ }^{\circledR}$, Diagnostic Product Corp., Los Angeles, CA). Plasma samples were stored at $-70{ }^{\circ} \mathrm{C}$ until assayed. Assay sensitivity was $0.01 \mathrm{ng} / \mathrm{ml}$. The intra- and interassay coefficients of variation (CV) were $2 \cdot 0 \%$ and $4 \cdot 5 \%$, respectively. Cortisol was assayed with a DPC Coat-a-count assay, a solid phase radioimmunoassay with a detection limit of $0.2 \mu \mathrm{g} / \mathrm{dl}$. The intraand interassay coefficients of variation (CV) averaged $5.0 \%$ and $8 \%$, respectively.

\section{Pulsatility and Statistical Analyses}

Analysis of GH pulsatility was performed by Cluster Program version $7 \cdot 0$ with a cluster size of $2 \times 2$ and a $t$ statistic of 3 and 2 for detecting significant increases and decreases in $\mathrm{GH}$, respectively. The mean pulse amplitude was calculated for each subject as the average of the cluster-defined peaks. Pulse frequency is the number of pulses detected by Cluster over a 24-h period. Twenty-four-hour mean GH was calculated as the average of all GH values over the 24 -h period. Nocturnal GH was calculated as the average of plasma GH values between 23:00 and 06:50 h.

Statistical analysis was performed using SAS version 6.12 (SAS Institute, Inc., Cary, NC). The differences in age and BMI among the different groups of subjects were assessed by Anova. The relationships between the different parameters of the $\mathrm{GH}$ profiles to the state of depression and the phase of the menstrual cycle were analysed using PROC GLM/linear regression with forward selection. Because of the effect of body weight on GH concentrations, all results were adjusted for BMI, which was introduced in the model as a covariate. Data are shown as mean $\pm \mathrm{SD}$. A value of $P \leq 0.05$ was selected to indicate statistical significance.

\section{Results}

Patients' descriptive and hormonal characteristics are shown in Table 1. BMI was similar in depressed patients and controls $(P=0 \cdot 14)$, and in the subjects studied during the follicular phase compared with the subjects studied in the luteal phase $(P=0 \cdot 87)$.

Twenty-four-hour mean GH concentrations were not different between depressed and control subjects $(P=0.93)$, and were not affected by the phase of the menstrual cycle $(P=0 \cdot 38)$. The introduction of BMI as a covariate did not change these results. As expected, there was an inverse relationship between 24 -h mean GH and BMI $(P=0 \cdot 0005)$.

There was no difference in $\mathrm{GH}$ pulse frequency between depressed women and healthy controls $(P=0 \cdot 95)$. Pulse frequency was higher during the follicular phase of the cycle $(P=0.032)$, and this relationship is not affected by depression $(P=0.46$ for the interaction of phase of menstrual cycle and depression state). Adjusting for BMI did not affect this relationship $(P=0 \cdot 028)$.

Nocturnal GH was higher in the follicular phase of the cycle whether it was the only variable $(P=0 \cdot 05)$ or whether total 24 -h $\mathrm{GH}$ was also introduced in the model as a covariate $(P=0.0138)$. The presence of depression did not affect this relationship $(P=0 \cdot 44)$ for the interaction of phase of the menstrual cycle and state of depression).

Representative GH profiles for each group are shown in Fig. 1.

Twenty-four-hour mean cortisol concentrations in women with depression was not different than in controls $(P=0 \cdot 97)$. It was also similar during the follicular and luteal phases of the menstrual cycle $(P=0 \cdot 73)$.

\section{Discussion}

Growth hormone secretion is sexually dimorphic. When women are compared to men, day-night differences are less prominent, 
Table 1. Hormonal and descriptive characteristics of depressed and control subjects recruited in the follicular and luteal phases of the menstrual cycle

\begin{tabular}{|c|c|c|c|c|c|}
\hline & \multicolumn{3}{|c|}{ Follicular } & \multicolumn{2}{|l|}{ Luteal } \\
\hline & Units & Depressed & Control & Depressed & Control \\
\hline Age & years & $29 \cdot 4(9 \cdot 8)$ & $28 \cdot 6(9 \cdot 7)$ & $28 \cdot 5(7 \cdot 4)$ & $30 \cdot 3(7 \cdot 4)$ \\
\hline BMI & $\mathrm{kg} / \mathrm{m}^{2}$ & $24 \cdot 9(5 \cdot 0)$ & $24 \cdot 5(5 \cdot 4)$ & $27 \cdot 1(5 \cdot 5)$ & $23 \cdot 5(3 \cdot 3)$ \\
\hline Estradiol & $\mathrm{pmol} / \mathrm{l}$ & $215 \cdot 7(128 \cdot 3)$ & $259 \cdot 6(168 \cdot 6)$ & $310 \cdot 7(231 \cdot 1)$ & $315 \cdot 7(164 \cdot 8)$ \\
\hline Progesterone & $\mathrm{nmol} / \mathrm{l}$ & $1 \cdot 5(0 \cdot 8)$ & $1 \cdot 6(0 \cdot 7)$ & $17 \cdot 6(13 \cdot 0)$ & $14 \cdot 7(12 \cdot 8)$ \\
\hline 24-h mean cortisol & $\mathrm{nmol} / \mathrm{l}$ & $256 \cdot 5(111 \cdot 5)$ & $199 \cdot 1(36 \cdot 5)$ & $207 \cdot 5(34 \cdot 7)$ & $251 \cdot 3(115 \cdot 8)$ \\
\hline 24-h mean GH & $\mathrm{ng} / \mathrm{ml}$ & $2 \cdot 6(1 \cdot 3)$ & $2 \cdot 8(1 \cdot 6)$ & $2 \cdot 4(1 \cdot 0)$ & $2 \cdot 9(1.5)$ \\
\hline Night $\mathrm{GH}^{*}$ & $\mathrm{ng} / \mathrm{ml}$ & $2 \cdot 4(1 \cdot 6)$ & $1 \cdot 9(1 \cdot 1)$ & $1 \cdot 2(0 \cdot 6)$ & $1 \cdot 6(0 \cdot 8)$ \\
\hline Total GH pulses ${ }^{* *}$ & & $8 \cdot 9(1.5)$ & $9 \cdot 7(1 \cdot 3)$ & $8 \cdot 8(1 \cdot 3)$ & $8 \cdot 3(1 \cdot 5)$ \\
\hline Average GH pulse amplitude & $\mathrm{ng} / \mathrm{ml}$ & $2 \cdot 6(1 \cdot 1)$ & $2 \cdot 6(1 \cdot 4)$ & $2 \cdot 5(1 \cdot 4)$ & $3 \cdot 35(2 \cdot 1)$ \\
\hline Average GH valley mean & $\mathrm{ng} / \mathrm{ml}$ & $0.21(0 \cdot 16)$ & $0.54(0.51)$ & $0.27(0.07)$ & $0 \cdot 31(0 \cdot 23)$ \\
\hline
\end{tabular}

Significant difference between the follicular and the luteal phases of the menstrual cycle ${ }^{\star} P=0 \cdot 047$.
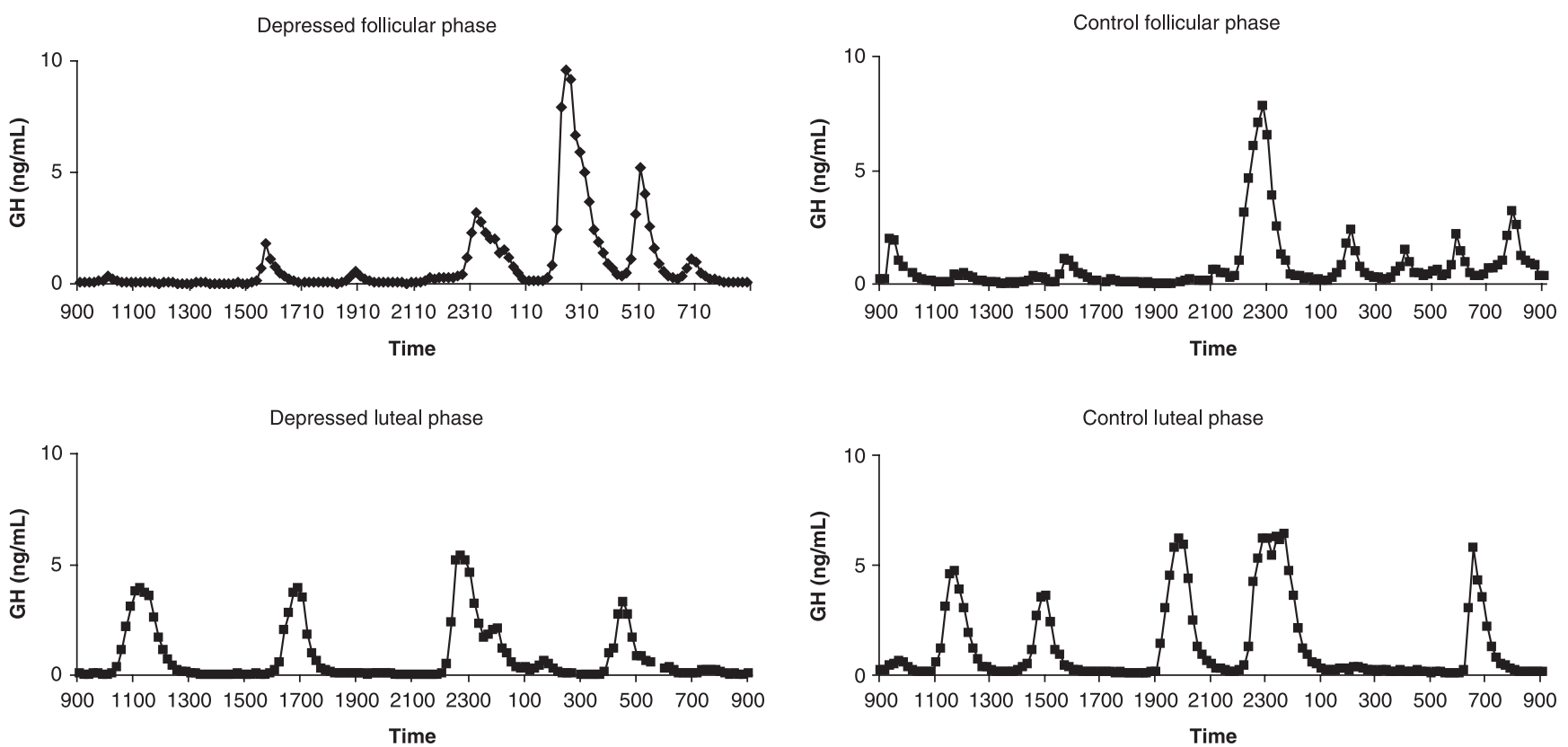

Fig. 1 Representative 24-h GH profiles for the follicular and luteal phases of the menstrual cycle.

interpulse baseline is higher, and the secretion pattern shows less orderliness. ${ }^{16}$ We studied $24-\mathrm{h}$ GH patterns in 19 women affected with depression, a condition with known endocrine disruptions of other pituitary-derived systems, ${ }^{19}$ and compared them to 24 normal controls. Our findings indicate that $24-\mathrm{h}$ GH secretion varies throughout the menstrual cycle with higher pulse frequency and an enhanced day-night variation during the follicular phase of the cycle. This difference between the follicular and luteal phases of the cycle was maintained regardless of depression.

Although GH-deficient states have been associated with depression, there have been conflicting reports regarding altered GH secretion in patients with established depression. Studies in women and late adolescent females have been limited either by the lack of control for the phase of the menstrual cycle, ${ }^{22}$ by a very small number of subjects $^{3}$ or insufficient sampling to establish a 24 -h profile. ${ }^{23}$ Variations of GH secretion during the menstrual cycle have been suggested, ${ }^{24}$ while others have found no differences between the follicular and luteal phases of the cycle. ${ }^{25}$ Other studies have only focused on differences of GH between the follicular and the periovulatory phases of the menstrual cycle, ${ }^{26}$ documenting a clear increase of GH pulses at mid-cycle. Several studies, ${ }^{11,27}$ have assessed $\mathrm{GH}$ response to pharmacological stimuli as an indirect mean for assessing GH dynamics, rendering inferences to endogenous diurnal rhythms difficult. Growth hormone concentrations are affected by weight and are lower in overweight and obese individuals. ${ }^{28}$ It is also known that GH decreases with ageing ${ }^{25}$ thus it is important to adjust for BMI and age and/or account for them in the selection of participants as was performed in our study. 
This is the first report on GH in women affected with depression and compared to controls matched for the phase of the menstrual cycle. As we have previously reported, depressed patients had lower estradiol levels in the follicular phase compared to controls; ${ }^{19}$ however, these depressed women did not demonstrate significantly increased cortisol concentrations when compared to their normal counterparts. In this study, depression had no significant impact on $\mathrm{GH}$ concentrations in either follicular or luteal phases of the menstrual cycle.

We used an unselected group of patients, including patients with atypical depression, rather than restricting our study to melancholic depressed women only. By recruiting unselected depressed women presenting to the psychiatric clinic to seek treatment, we found that only $19 \%$ of depressed women manifested increased baseline cortisol (greater than $9.7 \mu \mathrm{g} / \mathrm{dl}$ ). This is similar to the 7 of $32(22 \%)$ patients who had mean cortisol levels greater than $10 \mu \mathrm{g} / \mathrm{dl}$ in the study of Halbreich et al. ${ }^{29}$ The studies of Rubin et al. ${ }^{30}$ and Pfohl et al. ${ }^{31}$ used dexamethasone nonsuppression to define subgroups, and found normal baseline cortisol in the dexamethasone suppressors but significant increased cortisol secretion in dexamethasone nonsuppressors which were $15 / 40$ (38\%) patients in the study by Rubin et al. and 8/25 (32\%) in the study by Pfohl et al. We did not use dexamethasone to select a subgroup, but overall our data with women only agree with those studies including men and older female subjects showing normal cortisol secretion in most depressed subjects. Because of the quasi-normal cortisol level of our participants, there is still a possibility that patients with severe depression and markedly increased cortisol concentrations would show differences in $\mathrm{GH}$ concentrations when compared to normal subjects.

In summary, 24-h GH concentrations are maintained throughout the menstrual cycle. However, there is increased pulse frequency as well as enhanced nocturnal concentration in the follicular phase of the cycle. Depression does not affect these changes. We conclude that controlling for the menstrual cycle is needed in studies focused on $\mathrm{GH}$ secretion in women of reproductive age with or without depression.

\section{Acknowledgements}

We are grateful to Katie Aeshliman for technical assistance.

\section{References}

1 Young, E.A. \& Korszun, A. (2002) The hypothalamic-pituitarygonadal axis in mood disorders. Endocrinological Metabolism Clinical North America, 31 (1), 63-78.

2 Xiao, E., Xia-Zhang, L. \& Ferin, M. (2002) Inadequate luteal function is the initial clinical cyclic defect in a 12-day stress model that includes a psychogenic component in the Rhesus monkey. Journal of Clinical Endocrinological Metabolism, 87 (5), 2232-2237.

3 Jaffe, C.A., Ocampo-Lim, B., Guo, W. et al. (2000) Growth hormone secretory dynamics over the menstrual cycle. Endocrinology Journal, 47 (5), 549-556.

4 Lasser, R.A. \& Baldessarini, R.J. (1997) Thyroid hormones in depressive disorders: a reappraisal of clinical utility. Harvard Review of Psychiatry, 4 (6), 291-305.
5 Van den Berghe, G. (2002) Neuroendocrine pathobiology of chronic critical illness. Critical Care Cliny, 18 (3), 509-528.

6 Magiakou, M.A., Mastorakos, G., Gomez, M.T., Rose, S.R. \& Chrousos, G.P. (1994) Suppressed spontaneous and stimulated growth hormone secretion in patients with Cushing's disease before and after surgical cure. Journal of Clinical Endocrinological Metabolism, 78 (1), 131-137.

7 Sonino, N. \& Fava, G.A. (2001) Psychiatric disorders associated with Cushing's syndrome. Epidemiology, pathophysiology and treatment. CNS Drugs, 15 (5), 361-373.

8 Young, E.A., Lopez, J.F., Murphy-Weinberg, V., Watson, S.J. \& Akil, H. (2000) Hormonal evidence for altered responsiveness to social stress in major depression. Neuropsychopharmacology, 23 (4), 411418.

9 Young, E.A., Lopez, J.F., Murphy-Weinberg, V., Watson, S.J. \& Akil, H. (2003) Mineralocorticoid receptor function in major depression. Archives of General Psychiatry, 60 (1), 24-28.

10 Siever, L.J., Trestman, R.L., Coccaro, E.F. et al. (1992) The growth hormone response to clonidine in acute and remitted depressed male patients. Neuropsychopharmacology, 6 (3), 165-177.

11 Laakmann, G., Hinz, A., Voderholzer, U. et al. (1990) The influence of psychotropic drugs and releasing hormones on anterior pituitary hormone secretion in healthy subjects and depressed patients. Pharmacopsychiatry, 23 (1), 18-26.

12 Juul, A., Scheike, T., Pedersen, A.T. et al. (1997) Changes in serum concentrations of growth hormone, insulin, insulin-like growth factor and insulin-like growth factor-binding proteins 1 and 3 and urinary growth hormone excretion during the menstrual cycle. Human Reproduction, 12 (10), 2123-2128.

13 Ansseau, M., Von Frenckell, R., Cerfontaine, J.L. et al. (1988) Blunted response of growth hormone to clonidine and apomorphine in endogenous depression. British Journal of Psychiatry, 153, 65-71.

14 Checkley, S.A., Slade, A.P. \& Shur, E. (1981) Growth hormone and other responses to clonidine in patients with endogenous depression. British Journal of Psychiatry, 138, 51-55.

15 Lesch, K.P., Laux, G., Pfuller, H., Erb, A. \& Beckmann, H. (1987) Growth hormone $(\mathrm{GH})$ response to GH-releasing hormone in depression. Journal of Clinical Endocrinological Metabolism, 65 (6), $1278-1281$.

16 Jaffe, C.A., Ocampo-Lim, B., Guo, W. et al. (1998) Regulatory mechanisms of growth hormone secretion are sexually dimorphic. Journal of Clinical Investigation, 102 (1), 153-164.

17 Ovesen, P., Vahl, N., Fisker, S., Veldhuis, J.D., Christiansen, J.S. \& Jorgensen, J.O. (1998) Increased pulsatile, but not basal, growth hormone secretion rates and plasma insulin-like growth factor I levels during the periovulatory interval in normal women. Journal of Clinical Endocrinological Metabolism, 83 (5), 1662-1667.

18 Young, E.A., Carlson, N.E. \& Brown, M.B. (2001) Twenty-four-hour ACTH and cortisol pulsatility in depressed women. Neuropsychopharmacology, 25 (2), 267-276.

19 Young, E.A., Midgley, A.R., Carlson, N.E. \& Brown, M.B. (2000) Alteration in the hypothalamic-pituitary-ovarian axis in depressed women. Archives of General Psychiatry, 57 (12), 1157-1162.

20 First, M., Spitzer, R., Gibbon, M. \& Williams, J. (1994) Structured Clinical Interview for Axis 1 DSM-IV Disorders-Patient Edition (SCID-I/P, version 2.0). Biometrics Research Department, New York State Psychiatric Institute.

21 Spitzer, R.L., Williams, J.B., Gibbon, M. \& First, M.B. (1992) The structured clinical interview for DSM-III-R (SCID). I: history, rationale, and description. Archives of General Psychiatry, 49 (8), $624-629$. 
22 Franz, B., Kupfer, D.J., Miewald, J.M., Jarrett, D.B. \& Grochocinski, V.J. (1995) Growth hormone secretion timing in depression: clinical outcome comparisons. Biology Psychiatry, 38 (11), 720-729.

23 Kutcher, S., Malkin, D., Silverberg, J., Marton, P., Williamson, P., Malkin, A. et al. (1991) Nocturnal cortisol, thyroid stimulating hormone, and growth hormone secretory profiles in depressed adolescents. Journal of the American Academy of Child Adolescent Psychiatry, 30 (3), 407-414.

24 Bancroft, J., Cook, A., Davidson, D., Bennie, J. \& Goodwin, G. (1991) Blunting of neuroendocrine responses to infusion of 1-tryptophan in women with perimenstrual mood change. Psychological Medy, 21 (2), 305-312.

25 Zadik, Z., Chalew, S.A., McCarter, R.J. Jr, Meistas, M. \& Kowarski, A.A. (1985) The influence of age on the 24-h integrated concentration of growth hormone in normal individuals. Journal of Clinical Endocrinological Metabolism, 60 (3), 513-516.

26 Faria, A.C., Bekenstein, L.W., Booth, R.A. Jr et al. (1992) Pulsatile growth hormone release in normal women during the menstrual cycle. Clinical Endocrinology (Oxf), 36 (6), 591-596.
27 Laakmann, G., Munz, T., Hinz, A. \& Voderholzer, U. (1990) Influence of clenbuterol, a beta-adrenergic agonist, on desipramine induced growth hormone, prolactin and cortisol stimulation. Psychoneuroendocrinology, 15 (5-6), 391-399.

28 Rasmussen, M.H., Hvidberg, A., Juul, A. et al. (1995) Massive weight loss restores 24 -h growth hormone release profiles and serum insulin-like growth factor-I levels in obese subjects. Journal of Clinical Endocrinological Metabolism, 80 (4), 1407-1415.

29 Halbreich, U., Asnis, G.M., Shindledecker, R., Zumoff, B. \& Nathan, R.S. (1985) Cortisol secretion in endogenous depression. I. Basal plasma levels. Archives of General Psychiatry, 42 (9), 904908.

30 Rubin, R.T., Poland, R.E., Lesser, I.M., Winston, R.A. \& Blodgett, A.L. (1987) Neuroendocrine aspects of primary endogenous depression. I. Cortisol secretory dynamics in patients and matched controls. Archives of General Psychiatry, 44 (4), 328-336.

31 Pfohl, B., Sherman, B., Schlechte, J. \& Stone, R. (1985) Pituitaryadrenal axis rhythm disturbances in psychiatric depression. Archives of General Psychiatry, 42 (9), 897-903. 